\title{
Tumor neuroendocrino primario del hígado. Histopatología e inmunohistoquímica de tres casos confirmados por autopsia
}

\author{
Luis Carlos Mita-Albán ", Ana María Casco-Jarquín ², Rodrigo Álvarez-Ramírez ${ }^{3}$, José Alberto Ramírez-Corrales ${ }^{4}$.
}

\begin{abstract}
Resumen: El tumor carcinoide primario hepático (TCPH) es poco común. Existen pocos carcinoides del hígado descritos como primarios y menos aún, confirmados con una autopsia completa. De marzo de 2000 a abril de 2001, encontramos 3 casos de TCPH en fase terminal, con un patrón histológico atípico. Cada caso fue positivo, para una variedad amplia de marcadores inmunohistoquímicos. Todos ellos fueron confirmados por una autopsia. Estas neoplasias pueden ser detectadas por métodos diagnósticos confiables, como la excreción urinaria de ácido $5 \mathrm{OH}$-indolacético.
\end{abstract}

Descriptores: Hígado, carcinoide, neuroendocrino, inmunohistoquímica.

Recibido: 22 de enero, 2003

Aceptado: 01 de julio, 2003

Los tumores carcinoides son la segunda neoplasia más común del tracto gastrointestinal, después del adenocarcinoma ${ }^{1}$. De modo general, se caracterizan por tasas de crecimiento relativamente bajas, así como por pocas diseminaciones locales y generales comparadas con otras neoplasias malignas ${ }^{2}$. Se dispone de poca información en cuanto a patogenia, exceptuando los gástricos, para los cuales ha sido sugerido el rol de la hipergastrinemia ${ }^{3}$. En la mayoría de los casos, los síntomas se asocian con enfermedad metastásica, no obstante, esto no es la regla, Cooper y Smith reportaron 4 pacientes con síndrome carcinoide sin enfermedad metastási$\mathrm{ca}^{4}$. Todos ellos tenían masas retroperitoneales o intraabdominales drenando a la circulación sistémica. El TCPH es una neoplasia poco común, Sano y Makuuchi en su revisión mundial de bibliografía, informaron solamente 30 casos conocidos hasta $1999^{5}$. Existen pocos reportes confirmados por autopsia completa, ya que aún en casos de fallecimiento las autopsias pueden ser denegadas por los familiares ${ }^{6}$.

Abreviaturas: TCPH, tumor carcinoide primario hepático; CAP, campos de alto poder.

Médico Residente, Servicio de Patología . Hospital Calderón Guardia

Médico General, Hospital William Allen

Médico Residente, Servicio de Patología, Hospital México

Jefe de Servicio de Patología, Hospital Calderón Guardia

Correspondencia: Dr. Luis Carlos Mita Albán. mitaalban @ hotmail.com.

\section{Descripción de casos}

Caso 1 (A-00.147) Mujer de 43 años. Presentó astenia y dolores osteomusculares de 6 meses de evolución. Ingresó por cuadro de sangrado digestivo alto. En el examen físico se documentó hepatomegalia leve. El ultrasonido abdominal reveló múltiples lesiones hepáticas bien definidas. Falleció a los 20 días del ingreso, con diagnóstico clínico de "sepsis de origen pulmonar". En la autopsia se encontró un proceso bronconeumónico severo. El hígado, no cirrótico, pesó $1850 \mathrm{gm}$, tenía masas múltiples, en el lóbulo derecho, la mayor de 10 $\mathrm{cm}$. de diámetro y varias satélites de hasta $2 \mathrm{~cm}$, de diámetro, blanquecinas, firmes, sin necrosis, con escasos focos de hemorragia.

Caso 2 (A.00.167) Mujer de 77 años. Presentó dolor abdominal de 22 días de evolución con hiporexia y disnea. En el exámen físico de ingreso se palpó el hígado a $3 \mathrm{~cm}$ bajo el reborde costal derecho. El ultrasonido abdominal reveló múltiples lesiones nodulares hepáticas. La paciente presentó una evolución tórpida y falleció 18 días después de su ingreso. Hallazgos: El hígado pesó 4580 g y tenía un patrón nodular cirrótico. En el lóbulo derecho había un nódulo firme amarillento, de $8 \mathrm{~cm}$ y múltiples satélites hasta de $1 \mathrm{~cm}$ de diámetro (el mayor de ellos). Todos presentaban zonas centrales necróticas. Había infiltración de la cabeza del páncreas y masas pulmonares pequeñas en lóbulo superior derecho, con bronconeumonía concomitante. 
Caso 3 (A 00.91). Hombre de 67 años. Etilista activo. Hipertenso y diabético tipo II en tratamiento. Ingresó al Servicio de Emergencias del H.C.G, por cuadro de hematemesis. Por endoscopía se confirmó una várice subcardial sangrante. Se controló el cuadro inicial, pero resangró a las 72 horas de la primera endoscopía y falleció a los 3 días del ingreso. Se encontró un hígado de 2030 gramos, cirrótico, de color amarillento y consistencia aumentada; al corte se hallaron zonas reblandecidas y dos nódulos de $9 \mathrm{~cm}$ de diámetro cada uno, en el lóbulo derecho, con infiltración difusa del lóbulo izquierdo por múltiples satélites de hasta $4 \mathrm{~cm}$ de diámetro, con afección de entre un $75 \%$ y un $85 \%$ del parénquima hepático. Se documentó sangrado digestivo por várice subcardial y neumonía bibasal en hepatización roja. Histología e inmuno histoquímica en Cuadro 1. En la figura 1 se muestran aspectos micro y macroscópicos de las neoplasias.

\section{Discusión}

Los casos aquí reportados corresponden a neoplasias primarias del hígado, confirmadas todas por autopsia completa. La sintomatología presentada por estos pacientes es atípica en cuanto al patrón descrito para los tumores carcinoides, no obstante, esto es frecuente entre aquellos derivados del intestino anterior ${ }^{7}$.

El aspecto macroscópico de estos tumores en el hígado es variable, encontrándose casos descritos como primarios multinodales ${ }^{5,6,8,9}$, en tanto que otros autores describen casos de masa única ${ }^{10,11}$. El patrón histológico encontrado en estos tumores corresponde al descrito por Moyana ${ }^{12}$ para los carcinoides moderadamente diferenciados ( $\operatorname{casos} 1$ y 2 ) y pobremente diferenciados (caso 3). De acuerdo con Staren, nuestros dos primeros casos se clasificarían como carcinomas neuroendocrinos, del tipo célula intermedia y de célula pequeña, el último ${ }^{13}$. Las características histoquímicas e inmunohistoquímicas de estas neoplasias son también variables entre los informes de varios autores; el patrón típico de positividad para Grimelius, Enolasa Neuroespecífica, Cromogranina -A y de negatividad para Fontana Masson, en los derivados del intestino anterior, es reportado por la mayoría de ellos 5,6, 8,9,10,14. En nuestros casos, la tinción de Fontana Masson fue negativa en todos, lo cual es compatible con el patrón de los derivados de intestino anterior; fueron positivos para Cromogranina -A y Enolasa (en menor grado en el caso 3). La tinción por gastrina revela una positividad más extensa en estos casos complicados con sangrado digestivo, uno de ellos con una úlcera antral, cuyo examen histológico en múltiples cortes no reveló malformaciones vasculares, como varios autores han reportado ${ }^{15}$. La autopsia descartó la posibilidad de metástasis hacia el hígado de un carcinoide gástrico, como se han reportado en algunos casos ${ }^{16}$. La escasa sobrevida intrahospitalaria de nuestros tres pacientes es atribuible a lo avanzado de su enfermedad al momento del diagnóstico. En conclusión: entre marzo de 2000 y abril del 2001 encontramos tres casos de TCPH en estadios terminales con un patrón histológico no típico, el cual incluyó conteo de mitosis elevada. La asociación entre patrones arquitecturales histológicos y citológicos atípicos y conteos de mitosis elevados (más de dos mitosis en $10 \mathrm{CAP}$ ), fue descrita por Travis en sus series de carcinomas neuroendocrinos pulmonares ${ }^{17,18}$. No obstante, dado que desconocemos la evolución cronológica real de estos pacientes no nos es posible correlacionar, en nuestros casos, el conteo de mitosis con la agresividad clínica. La sintomatología de los casos fue, a todas luces, atípica ${ }^{19}$. La probabilidad de tumor neuroendocrino no fue planteada clínicamente en ninguno de los tres. Tal baja sospecha diagnóstica es atribuible a la poca frecuencia de estas neoplasias 5,6,8-10,14,19. Las neoplasias pueden ser detectadas por métodos diagnósticos rápidos y confiables, como la excreción en orina de 24 horas de ácido $5 \mathrm{OH}$ - Indol acético, con tal prueba se obtiene entre un $65 \%$ y un $75 \%$ de sensibilidad y un 90 $\%$ y un $100 \%$ de especificidad ${ }^{20}$. Por último, la realización de autopsia, y no otros procedimientos diagnósticos premortem, permitió identificar los primarios hepáticos, lo cual recalca una vez más la importancia de la autopsia como generadora de conocimiento.

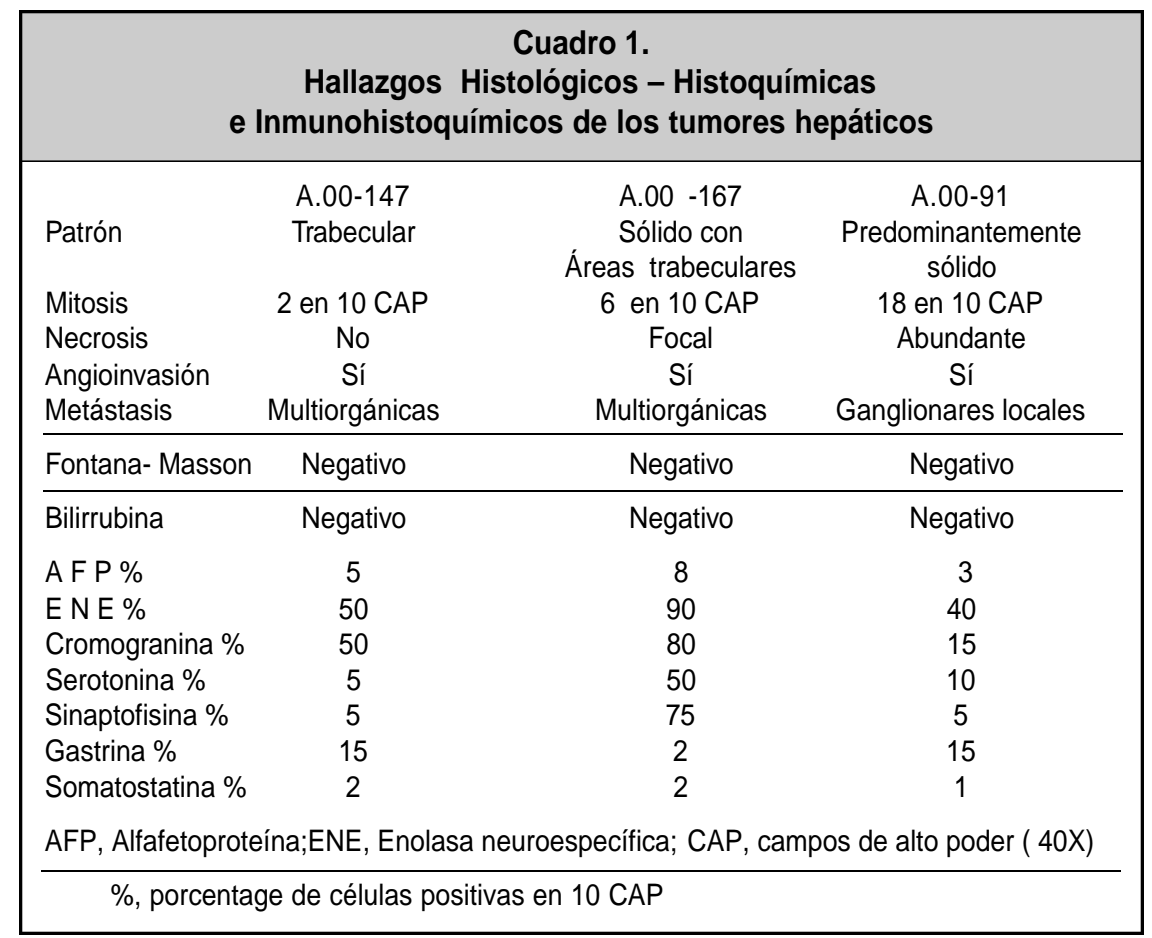

114 AMC, Julio-Setiembre 2003, vol 45 (3) 

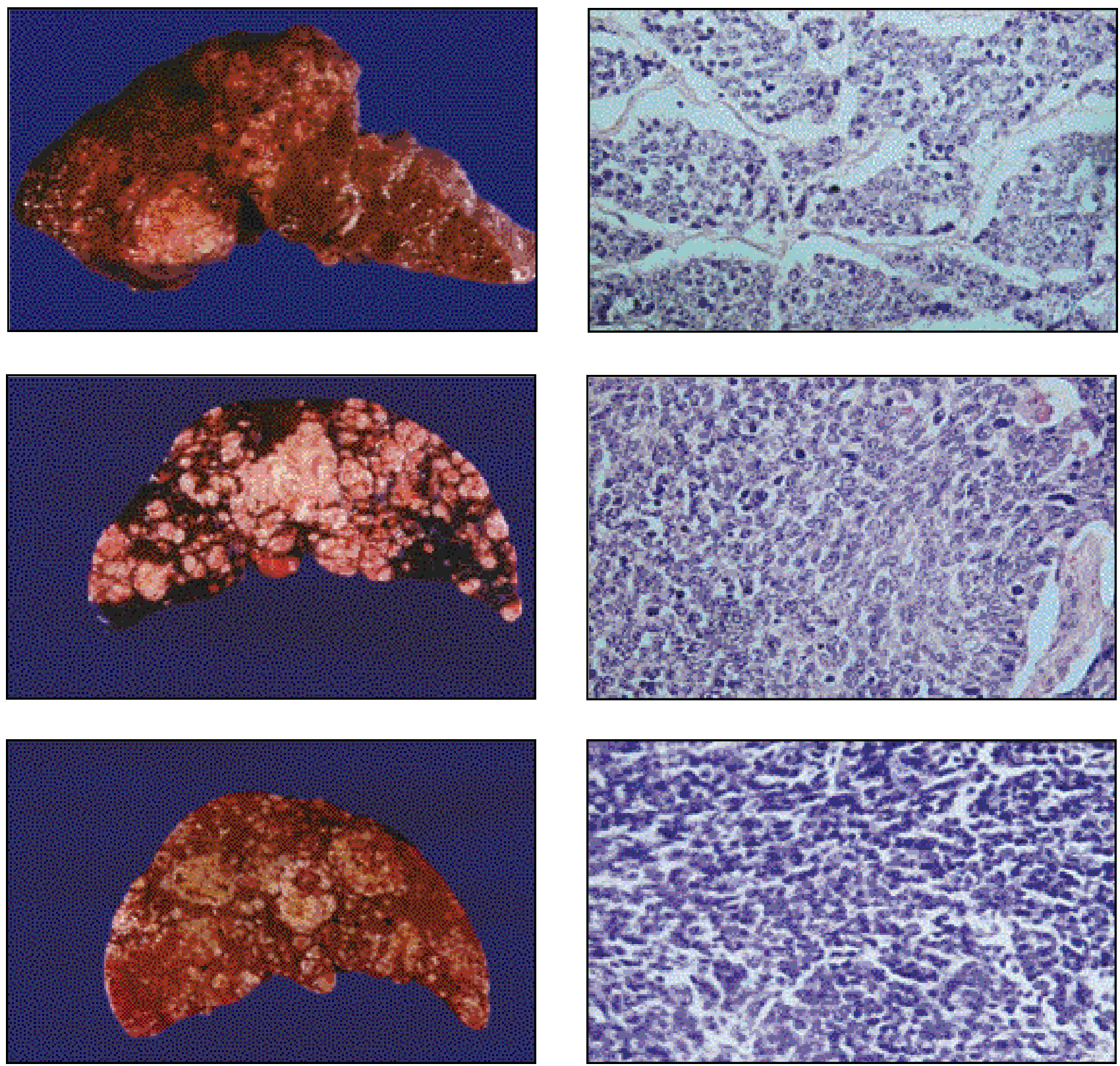

Figura 1. Aspectos macro y microscópico ( $40 \mathrm{x}$ ) de las neoplasias hepáticas. A: Masa dominante bien delimitada en lóbulo derecho. Nidos organoides y trabéculas, rodeadas de trama capilar fina ,con células de citoplasma amplio y ocasionales figuras de mitosis B: Masas dominantes en lóbulo derecho y extensa infiltración del parénquima .Células de citoplasma amplio con mayor pleomorfismo y alteración de la relación núcleo citoplasma .C:Masas en lóbulo derecho e izquierdo con múltiples satélites. Las células tienen citoplasma escaso y núcleos amplios. 


\section{Abstract}

A primary hepatic carcinoid tumor (PHCT) is an uncommon finding. There are few reports of primary hepatic carcinoid tumors, and less confirmed with a complete autopsy. From March 2000 to April 2001 we found 3 cases of PHCT in terminal phase with an atypical histologic pattern . Each case was positive to a wide variety of inmuno histochemical markers . All of them were confirmed by an autopsy. These neoplasies can be detected by reliable diagnostic tools such as the urinary excretion of $5 \mathrm{OH}$ indolacetic acids.

\section{Referencias}

1. Angeletti S, Annibale B ,Marignani M,Corleto VD . Natural history of intestinal carcinoids. Ital J Gastroenterol Hepatol 1999; 31 Suppl 2: S108-10.

2. Shebani KO ,Souba WW , Finkelstein DM, Ott MJ . Prognosis and survival in patients with gastrointestinal tract carcinoiod tumors. AnnSurg 1999; 229:815-823.

3. Vinik A, McLeod MK, Shapiro B , Lloyd RV . Clinical features, diagnosis and localization of carcinoid tumors and their management. Gastroenterol Clin North Am 1989;18:865-893.

4. Cooper MA , Smith A, Khalifa M . Carcinoid syndrome from gastrointestinal carcinoid tumor without distant metastases. J Clin Gastroenterol 2002; 35 : 106-107.

5. Sano K, Kosuge T, Yamamoto J, Makuuchi M . Primary hepatic carcinoid tumors confirmed with long -term follow up after resection. Hepatogastroenterology 1999; 46 suppl 28: 2547-2550.

6. Andreola S, Lombardi L, Audisio RA, Makowka L, Starzl TE, VanThiel DH . A clinicopathologic study of primary hepatic carcinoid tumors . Cancer 1990 ; $65: 1211-1218$.

7. Caplin ME, Buscombe JR, Hilson AJ, Burroughs AK . Carcinoid Tumour. Lancet $1998 ; 352:$ 799-805.

8. Asakawa T , Tomioka T ,Abe K ,Kanematsu T. Primary hepatic carcinoid tumor. J Gastroenterol 1999; 34 : 123 -127.
9. Miura K ,Shirasawa H . Primary carcinoid tumor of the liver. Am J Clin Pathol 1988; 89: 561-564.

10. Moriura S, Ikeda S , Gotou S . Hepatic gastrinoma. Cancer 1993; 72 : 1547-50.

11. Ali M, Fayemi AO, Braun EV. Malignant apudoma of the liver with symptomatic intractable hypoglicemia. Cancer 1978; 42:686-692.

12. Moyana TN ,Xiang J ,Senthilselvan A, Kulaga A . The spectrum of neut roendocrine differentiation among gastrointestinal carcinoids .Arch Pathol Lab Med 2000; 124 :570 -576.

13. Staren ED, Gould VE, Warren WH, Wool NL,Economou SG. Neuroendocrine carcinomas of the colon and rectum:A clinicopathologic evaluation. Surgery 1988; 104: 1080-1089.

14. Travis WD, Gal AA, Colby T. Reproducibility of neuroendocrine lung tumor classification. Hum Pathol 1998; 29: 272-279.

15. Travis WD , Rush W, Koss MN . Survival analysis of 200 pulmonary neuroendocrine tumors with clarification of criteria for atypical carcinoid and its separation from typical carcinoid. Am J Surg Pathol 1998; 22: 934-944

16. Travis WD, Beasley MB, Thunnissen F ; Brambilla E, Steele R . Pulmonary atypical carcinoid: Predictors of survival in 106 cases. Hum Pathol $2000 ; 31: 1255-1265$.

17. Sioutos N, Virta S, Kessimian N . Pirmary hepatic carcinoid tumor. An electron microscopic and immunohistochemical study. Am J Clin Pathol 1991; 95: 172. 175.

18. Ronconi L, Costi R, Canavese G, Bordi C . Carcinoid tumor associated with vascular malformation as a cause of massive gastric bleeding. Am J Gastroenterol 1997; 92:2119-2120?

19. Schwarz RE,Fuller RA ,Chu P. Gastric carcinoid metastatic to the liver. J Clin Oncol 2002, 20: 2403-2404.

20. Modlin IM , Sandor A . An analysis of 8305 cases of carcinoid tumors. Cancer 1997; 79: 813-829.

21. Tomassetti P. Clinical aspects of carcinoid tumours. Ital J Gastroenterol Hepatol 1999; 31 suppl 2: S 143-146.

\section{AMC, Julio-Setiembre 2003, vol 45 (3)}

